\title{
ESTÉTICA ABSTRACTA GEOMÉTRICA DE LOS CAZADORES RECOLECTORES DEL ÁREA DE VENTANIA IPROVINCIA DE BUENOS AIRES, REPÚBLICA ARGENTINA)
}

\section{ABSTRACT GEOMETRIC AESTHETICS OF THE VENTANIA HUNTER-GATHERERS (BUENOS AIRES PROVINCE, ARGENTINA)}

\author{
María CeCIIIA PanizZA*
}

El arte rupestre constituye un sistema de comunicación visual, en el cual cada motivo estaría integrando un signo con un determinado significado simbólico, al mismo tiempo que se habrían utilizado ciertas reglas para combinarlos en patrones reconocibles para las sociedades responsables de su ejecución y uso. En la región pampeana argentina esta materialidad se caracteriza por el predominio de los motivos rectilíneos, generalmente de color rojo, englobados en una tendencia estilística abstracta de modalidad geométrica. En este trabajo se considera el conjunto de las representaciones rupestres de los grupos cazadores recolectores que habitaron en el pasado en el área del sistema serrano de Ventania y su llanura adyacente durante el Holoceno Tardío (actual provincia de Buenos Aires, República Argentina), y su estudio se aborda desde una perspectiva integradora de las cualidades estéticas de las representaciones en el marco de la semiótica visual.

Palabras clave: estética, semiótica, arte rupestre abstracto geométrico, región pampeana, Ventania, cazadores recolectores

Rock art is a visual communication system in which each motif constitutes a sign with a particular symbolic meaning and certain rules are used to combine those signs into patterns recognizable to the societies that created and used them. In the Pampean Region of Argentina this materiality is characterized by the predominance of rectilinear motifs, usually red in color and in an abstract geometric style. This work examined the cave representations of ancient bunter-gatherer groups that lived during the late Holocene in the area of Ventania and its adjacent plain (present-day Buenos Aires Province, Argentina). The motifs are studied from a comprehensive perspective that considers the aesthetic qualities of the representations in the framework of visual semiotics.

Key words: aesthetics, semiotics, abstract geometric rock art, Pampean Region, Ventania, bunter-gatherers

\section{INTRODUCCIÓN}

Cada sociedad humana posee sistemas compartidos de símbolos, valores y creencias, propios e inherentes, y desde la arqueología se considera cómo estos aspectos simbólicos y rituales inciden en la formación de la cultura material. Una de las manifestaciones más antiguas y conocidas del hombre es el arte rupestre, un fenómeno que ha sido estudiado desde diversas disciplinas -historia del arte, filosofía del arte, artes plásticas, psicología social, antropología y arqueología-, con distintos enfoques y metodologías. Este trabajo se enfoca sobre dos características básicas de este fenómeno cultural: como materialidad estética y comunicativa al mismo tiempo, lo que permite abordarlo desde una perspectiva estética y semiótica (Eco 1995 [1976]; Dettwiler 1986). En este sentido, las representaciones rupestres serían el producto material de un sistema semiótico guiado por un criterio estético de una sociedad en un contexto histórico y espacial determinados (Troncoso 2005).

En la región pampeana argentina, el fenómeno expresivo conocido como arte rupestre ha sido descrito en tres zonas acotadas que corresponden a ambientes ecológicos distintos: las serranías de Tandilia (pampa húmeda) y Ventania (Área Ecotonal Húmedo-Seca Pampeana), en la provincia de Buenos Aires y al oeste en la provincia de La Pampa (pampa seca). Se considera que el área del sistema serrano de Ventania constituye un

\footnotetext{
* María Cecilia Panizza, Becaria Consejo Nacional de Investigaciones Científicas y Técnicas (CONICET), Centro de Estudios Arqueológicos Regionales (Facultad de Humanidades y Artes, Universidad Nacional de Rosario), Argentina, email: mcpanizza@yahoo.com.ar
} 
espacio destacable para el desarrollo de estudios sobre representaciones visuales, por la cantidad de cuevas y abrigos rocosos que presentan pinturas, y por el hallazgo de sitios arqueológicos con materiales simbólicos en su llanura adyacente, como placas grabadas, cerámica decorada, entre otros elementos. En esta oportunidad, el arte rupestre es analizado desde el punto de vista estético (a partir de su materialidad) y desde una perspectiva semiótica (vinculado a la producción de significado). Entre el conjunto de las representaciones se seleccionaron aquellas de carácter abstracto-geométrico, las cuales conformarían una modalidad estética particular y predominante durante el Holoceno Tardío.

\section{ÁREAS Y MATERIALES DE ESTUDIO}

Los sitios arqueológicos considerados en la presente investigación se encuentran ubicados en distintos sectores del sistema serrano de Ventania, en el sur del Área Ecotonal Húmedo-Seca Pampeana (Oliva \& Algrain 2005), en una zona de ecotono entre las regiones de pampa húmeda y de pampa seca. Estas serranías se emplazan en una estructura con forma de arco, de rumbo general nortesureste, y sus dimensiones máximas son de $180 \mathrm{~km}$ en dirección NNO-SSE y de aproximadamente 130 km E-O (Schiller 1930). Morfoestructuralmente se diferencian dos sectores (Suero 1972): el occidental, que comprende las sierras de Puan, Curamalal, Bravard y Ventana, y el oriental, que abarca las sierras de Las Tunas y de Pillahuincó, con un menor grado de plegamiento asociado.

En este espacio destacan las cuevas como lugares con mayor estabilidad temporal, lo cual, junto con la proximidad a recursos considerados críticos para los grupos cazadores recolectores que lo habitaron (como materia prima lítica, cursos de agua, entre otros), habría favorecido su reutilización frecuente. En este sentido, Oliva (2000) propone que el sistema serrano de Ventania habría actuado como un factor concentrador de grupos humanos a través del tiempo.

Entre las numerosas cuevas y aleros existentes dentro del sistema serrano de Ventania se han detectado hasta el presente treinta y dos sitios que poseen en sus soportes representaciones rupestres (fig. 1). Estas representaciones

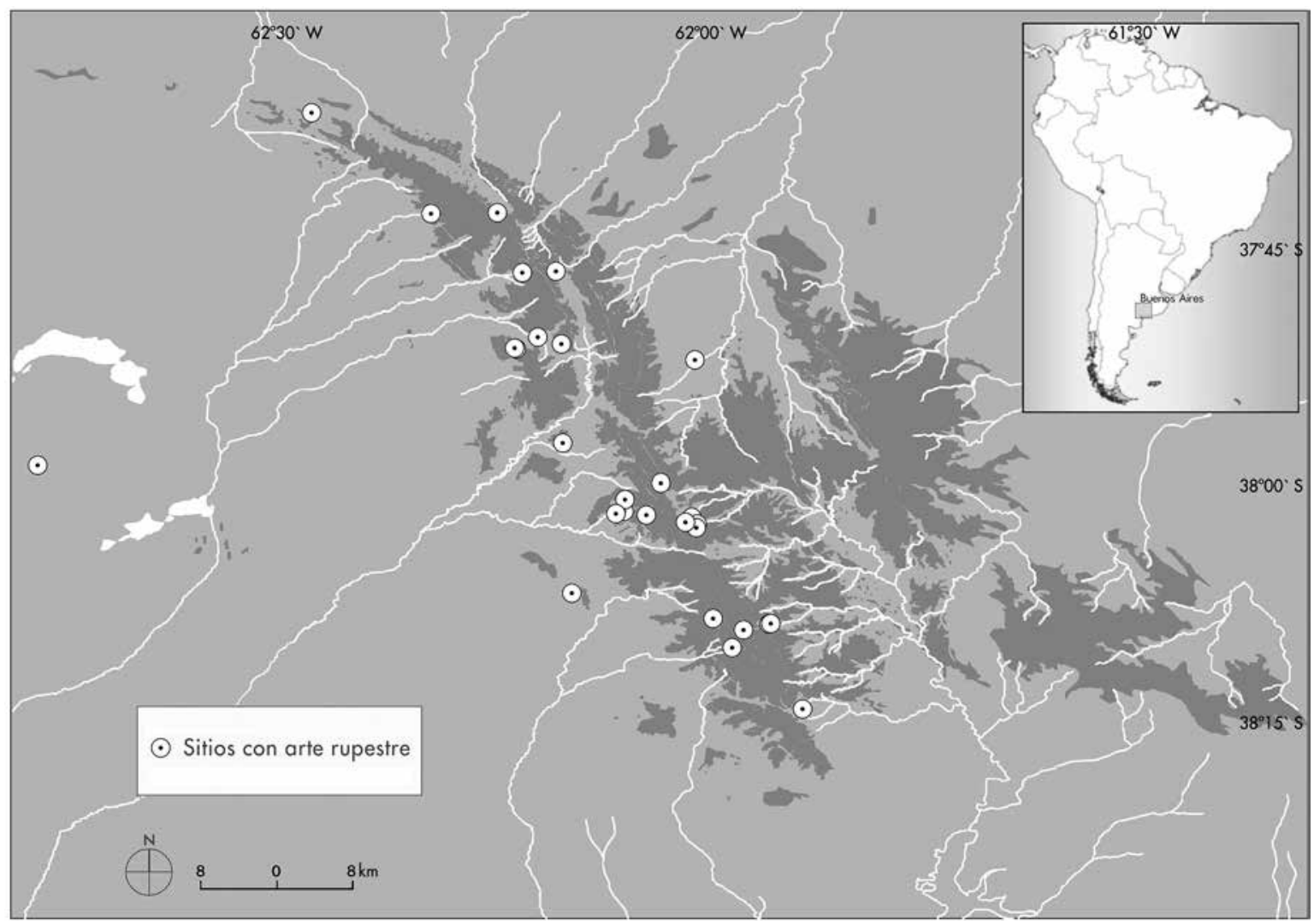

Figura 1. Mapa del área de Ventania (correspondiente al sudoeste de la provincia de Buenos Aires, República Argentina), donde se observa la distribución de los sitios con representaciones rupestres.

Figure 1. Map of the Ventania area (southeast sector of Buenos Aires Province, Argentina), showing the distribution of sites with rock art representations. 
consisten, en todos los casos, de pinturas en las cuales predomina el color rojo, aunque también se han hallado pinturas naranja y amarillo-ocre. En treinta y un sitios se han documentado motivos que formarían parte de la llamada tendencia abstracta de modalidad geométrica, enunciada por Gradín (1997-1998) para La Pampa y la Patagonia, y reformulada por Podestá y colaboradores (2011), y que constituyen la materia de análisis del presente trabajo. Sin embargo, es necesario mencionar los escasos motivos figurativos que se registran en siete de los sitios: antropomorfos y mascariformes en Gruta de los Espíritus (valle intraserrano Cueva 1), manos en positivos en Santa Marta 1, un barco y una mano en Cueva Florencio, un mascariforme en Cerro Cortapiés, un antropomorfo en Arroyo Concheleufú Chico, y biomorfos en los sitios La Montaña 3 y La Sofía 1 (Pérez Amat et al. 1985; Madrid \& Oliva 1994; Consens \& Oliva 1999; Oliva et al. 2010).

Considerando el número de las representaciones, su dispersión en el área de estudio y su homogeneidad técnica y morfológica, este análisis se centra exclusivamente en los motivos clasificados como geométricos abstractos correspondientes a treinta y uno de los treinta y dos sitios identificados en total (Tabla 1): Abra Agua Blanca 1, Arroyo Concheleufú Cueva 1, Arroyo San Bernardo Cuevas 1 y 2, Arroyo San Pablo Cueva 1, Cerro Cortapiés Cueva 1, Cueva Cerro Manitoba, Cerro Tres Picos Alero 1 y Cueva 2, Alero Corpus Christi, Cueva del Toro, Cueva Florencio, Hogar Funke Cueva 1, La Montaña 3, La Sofía 1, 2, 3, 4, 6 y 7 (o Valle Intraserrano Cueva 3), Parque Tornquist Cuevas 1, 2, 3, 4 y 5, Santa Marta 1, 2, 3, 4 y 5; y Valle Intraserrano Cueva 2. En primer lugar, se describen las cualidades estéticas tanto de las pinturas como de las cuevas y aleros rocosos, para luego desarrollar las propiedades semióticas del arte rupestre en tres niveles (marcas, atractores y operaciones combinatorias empleadas). Posteriormente, se realiza una comparación con la estética presente en elementos simbólicos portátiles del área de estudio y, a modo de conclusión una síntesis de los resultados alcanzados hasta el momento.

\section{Las cualidades estéticas del arte rupestre de Ventania}

La estética puede definirse como la capacidad humana y la tendencia a unir las propiedades cualitativas a un estímulo, y su estudio es importante para entender por qué las personas se comportan como lo hacen (Morphy 1993), en otras palabras, tiene que ver con la percepción, apreciación y comprensión.

Específicamente, la estética del arte rupestre abarca el estudio de las marcas grabadas o pintadas hechas por el hombre sobre una superficie rocosa como objetos de percepción, e incluye los diversos aspectos relevantes para la apreciación de una imagen de arte rupestre, en determinados contextos culturales y localizaciones geográficas, cuando son la base para experiencias perceptuales particulares (Heyd 2005).

La apreciación estética implica la valoración de algo, ya que cuando algo es apreciado se le atribuye un cierto valor; el enfoque está orientado a las cualidades formales de ese objeto, su composición y la manera en que se relacionan su forma y su contenido (Heyd 2001), además de los efectos que busca crear sobre el observador.

Las cualidades estéticas están vinculadas con la capacidad de ciertos objetos de atraer la atención e impactar sensorialmente más que otros, el valor que se les asigna a estos objetos deriva en parte del impacto que provocan. Morphy (1993) divide en dos partes la reacción estética: una más objetiva y universal relacionada con la percepción de las características físicas de un objeto (o imagen en este caso), y otra vinculada con la relación de esas características al conjunto subjetivo de connotaciones culturales.

A continuación se señalan las propiedades estéticas de las representaciones rupestres del área de Ventania. Si se concibe el sitio arqueológico (en este caso son cuevas o aleros rocosos) como escenario, se puede considerar la disposición de las pinturas como disposición de la obra, e indagar datos sobre el espectador al que está dirigido el mensaje (si este es público o privado, si las pinturas están ocultas o no). En el área relevada existen cientos de cuevas y aleros donde pudieron ejecutarse estas representaciones y, sin embargo, solo algunas fueron seleccionadas para servir de escenarios para estas pinturas, exclusivamente en el sector occidental del sistema serrano de Ventania, en las cuencas superiores de los ríos Sauce Chico y Sauce Grande, y de los arroyos Chasicó, 27 de Diciembre, Curamalal Grande y Napostá. Cabe destacar que tampoco usaron ningún basamento rocoso a cielo abierto para hacer representaciones al aire libre (o no se han conservado). En este sentido, estos lugares presentarían características valoradas o que han producido algún tipo de efecto sobre los antiguos habitantes de la sierra o grupos de cazadores recolectores que las ejecutaron, que los llevó a seleccionar estos lugares y no otros para dejar su marca en el soporte rocoso.

Entre otras características físicas de los sitios estudiados, algunas abordadas en Oliva (2000), podemos mencionar que estos se encuentran orientados principalmente en dos direcciones: entre el noreste y el noroeste, y entre el sur y sudeste, lo cual está relacionado con la formación, la estructura y las diferentes intensidades 
Tabla 1. Marcas, atractores y operaciones combinatorias definidas por cada sitio con representaciones rupestres Table 1. Details of marks, attractors and combining operations for each site with rock art representations

\begin{tabular}{|c|c|c|c|}
\hline \multirow{2}{*}{$\begin{array}{c}\text { Sitios con arte rupestre } \\
\text { Nombre }\end{array}$} & \multicolumn{3}{|c|}{ Semiótica } \\
\hline & Marcas & Atractores & Operaciones combinatorias \\
\hline Abra Agua Blanca 1 & punto, línea recta, $\mathrm{V}$ & no icónico & repetición, combinación simple \\
\hline Arroyo Concheleufú Cueva 1 & línea recta, rectángulo & icónico y no icónico & repetición, combinación simple \\
\hline Arroyo San Bernardo Cueva 1 & línea recta & no icónico & repetición, combinación simple \\
\hline Arroyo San Bernardo Cueva 2 & línea recta & no icónico & repetición \\
\hline Arroyo San Pablo Cueva 1 & línea recta, quebrada & no icónico & repetición \\
\hline Cerro Cortapiés Cueva 1 & línea recta, elipse, punto & icónico y no icónico & repetición, concentricidad \\
\hline Cueva Cerro Manitoba & $\begin{array}{c}\text { línea quebrada u ortogonal, } \\
\text { linea recta, V, zigzag, rombo, } \\
\text { triángulo }\end{array}$ & no icónico & repetición, combinación simple, superposición \\
\hline Cerro Tres Picos Alero 1 & línea recta & no icónico & repetición \\
\hline Cerro Tres Picos Cueva 2 & $\begin{array}{c}\text { línea recta, quebrada, } \mathrm{V} \text {, } \\
\text { zigzag }\end{array}$ & no icónico & repetición, combinación simple, superposición \\
\hline Corpus Christi Alero & $\begin{array}{l}\text { línea recta, zigzag, quebrada, } \\
\text { triángulo }\end{array}$ & no icónico & repetición, combinación simple, superposición \\
\hline Cueva del Toro & línea recta & no icónico & repetición \\
\hline Cueva Florencio & $\begin{array}{l}\text { línea recta, punto, línea } \\
\text { quebrada }\end{array}$ & icónico y no icónico & repetición, combinación simple, superposición \\
\hline Hogar Funke Cueva 1 & línea recta & no icónico & repetición \\
\hline La Montaña 3 & línea recta, quebrada, punto & icónico y no icónico & repetición, combinación simple, superposición \\
\hline La Sofía 1 & $\begin{array}{l}\text { línea recta, V, quebrada, línea } \\
\text { curva }\end{array}$ & icónico y no icónico & repetición, combinación simple \\
\hline La Sofía 2 & línea recta & no icónico & repetición \\
\hline La Sofía 3 & línea recta & no icónico & ninguna \\
\hline La Sofía 4 & línea recta & no icónico & repetición \\
\hline La Sofía 6 & línea recta & no icónico & repetición \\
\hline $\begin{array}{l}\text { La Sofía } 7 \text { o Valle Intraserrano } \\
\text { Cueva } 3\end{array}$ & línea recta, triángulo & no icónico & $\begin{array}{c}\text { repetición, combinación simple, superposición, } \\
\text { simetría bilateral }\end{array}$ \\
\hline Parque Tornquist Cueva 1 & triángulo, zigzag & icónico y no icónico & repetición, combinación simple \\
\hline Parque Tornquist Cueva 2 & $\begin{array}{l}\text { líneas quebradas, zigzag, } \\
\text { rombo, triángulo lleno, línea } \\
\text { recta }\end{array}$ & no icónico & repetición, combinación simple, superposición \\
\hline Parque Tornquist Cueva 3 & línea recta & no icónico & repetición \\
\hline Parque Tornquist Cueva 4 & línea recta & no icónico & ninguna \\
\hline Parque Tornquist Cueva 5 & línea recta & no icónico & repetición \\
\hline Santa Marta 1 & línea recta, rectángulo & icónico y no icónico & repetición, combinación simple \\
\hline Santa Marta 2 & línea recta & no icónico & ninguna \\
\hline Santa Marta 3 & $\begin{array}{l}\text { línea recta, zigzag, } \\
\text { subrectangular }\end{array}$ & no icónico & repetición, superposición \\
\hline Santa Marta 4 & $\begin{array}{l}\text { línea recta, punto, zigzag, } \\
\text { triángulo lleno }\end{array}$ & no icónico & repetición, superposición, combinación simple \\
\hline Santa Marta 5 & línea recta & no icónico & repetición, combinación simple \\
\hline Valle Intraserrano Cueva 2 & línea recta & no icónico & repetición \\
\hline Valle Intraserrano Cueva 1 & $\begin{array}{l}\text { rectángulo, elipse, punto, } \\
\text { triángulo, línea recta, } \\
\text { ortogonal }\end{array}$ & icónico & $\begin{array}{l}\text { repetición, combinación simple, superposición, } \\
\text { simetría bilateral, concentricidad }\end{array}$ \\
\hline
\end{tabular}


de plegamiento que originaron las cuevas en el sistema serrano. Además, la mayoría de las cuevas estudiadas se localizan en alturas similares entre los 550 y $650 \mathrm{msnm}$, excepto diez que se ubican por debajo de los $550 \mathrm{msnm}$, y solo una por encima, a los $715 \mathrm{msnm}$. Por otra parte, seis de los sitios se encuentran entre 800 y $1000 \mathrm{~m}$ de distancia de una fuente fluvial; en tanto los 26 restantes están localizados entre los 50 y 150 m de los cursos de agua más próximos. Si se comparan estas distancias con las alturas de los sitios, se observa que las cuevas que se localizan a menores alturas son las que están a mayores distancias de los cursos de agua; en cambio, las ubicadas a mayores alturas están muy próximas a cursos de agua.

En el área de Ventania, los motivos rupestres fueron realizados con pintura, en una gama cromática restringida, que incluye distintas tonalidades rojizas y unas pocas pinturas en coloraciones naranja y amarillo-ocre, en paredes y techos de cuevas y aleros. En la mayoría de las cuevas se encuentran motivos en rojo desvaído; en once de las cuevas se observan algunos motivos en rojo oscuro; en dos cuevas se observan motivos en rojo violáceo; en una cueva aparece también un motivo en ocre amarillo; y en otro sitio se ve una representación en color anaranjado. Cabe destacar, como característica técnica, el amplio uso de las concavidades naturales del sustrato rocoso para aplicar pintura.

Respecto de la visibilidad, la mayoría de las representaciones no están ocultas (70\%), por lo cual, son de fácil reconocimiento. Solo en dos de las cuevas aparecen motivos semiocultos (8\%) y en una aparecen algunos ocultos (4\%), mientras que en dos sitios se observan motivos no ocultos y semiocultos ( $8 \%$ ), y en otros dos sitios motivos ocultos y semiocultos (8\%).

La percepción de estas representaciones se realiza principalmente a través del sentido de la vista. A partir de las condiciones de acceso al sitio (cueva o alero), de la visibilización (campo visual que se abarca desde el sitio) y de la visibilidad (capacidad de ser observado desde otros lugares), además de la visibilidad de la representación rupestre dentro del sitio, es posible mencionar dos tipos de destinatarios:

1. para todos: el sitio es visible y accesible, al igual que las representaciones rupestres ejecutadas en el sitio. No hay restricción en su acceso a la experiencia. El mensaje que se transmite es público. Dos ejemplos son los sitios Arroyo Concheleufú Chico Cueva 1 y Cueva Florencio. Se supone que estas pinturas fueron realizadas para ser observadas, y en ese caso, las poblaciones volverían al mismo lugar para reeditar, para volver a vivir la experiencia que generaba su contemplación.

2. para unos pocos: existe algún tipo de dificultad para alcanzar la experiencia que involucran las pinturas. a) En este caso, el acceso al sitio es complicado, pero una vez en el sitio, las pinturas son fácilmente observables. El mensaje que se transmite a través de las representaciones es de contenido semipúblico o semiprivado. En este sentido, se puede nombrar como referencia el sitio Parque Tornquist Cueva 2 (fig. 2). Hay que considerar también que algunas veces existe más de un camino para llegar al sitio, por ejemplo, en este caso, se puede subir por el cañadón, que es una vía de acceso difícil, pero más fácil de visualizar el sitio, o se puede descender desde la cumbre, pero desde esa posición es extremadamente difícil visualizarlo. b) Esta categoría abarca aquellos sitios que pueden ser accesibles o no, pero las representaciones están ubicadas en lugares ocultos, por lo tanto solo aquellos que poseen el conocimiento previo de su presencia pueden observarlas. El mensaje que se transmite a

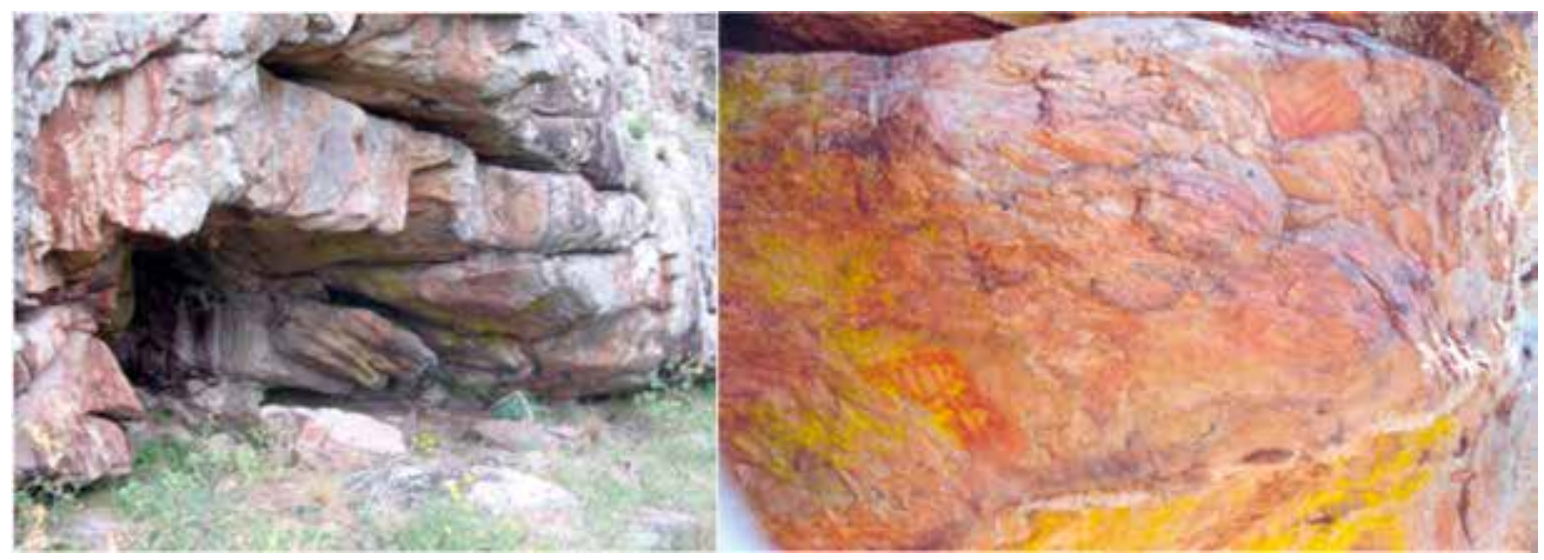

Figura 2. Vista del sitio y pinturas abstractas geométricas en Cueva Parque Tornquist 2.

Figure 2. View of the site and abstract geometric paintings of Cueva Parque Tornquist 2. 


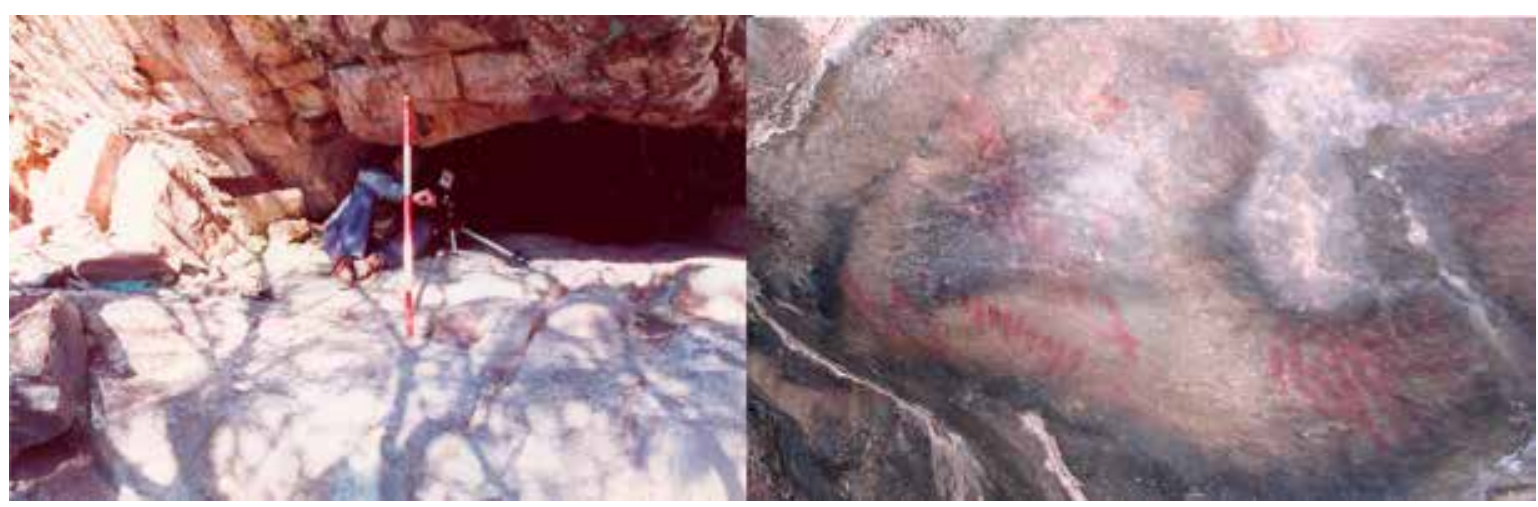

Figura 3. Vista del sitio y pinturas abstractas geométricas que se observan en el techo de Cueva Cerro Manitoba. Figure 3. View of the site and abstract geometric paintings observed on the ceiling of Cueva Cerro Manitoba.

través de la pintura es privado. Cumpliendo con las características mencionadas, se puede citar el sitio Cueva Cerro Manitoba (fig. 3).

$\mathrm{El}$ arte rupestre como evidencia cultural es el resultado de la experiencia estética tanto del ejecutante en el pasado como del observador en el presente, como productor de efectos emotivos y agente de transformación estética de la realidad. La estética es una reflexión que se hace sobre objetos y que produce un juicio estético (ideas producidas a partir de la percepción sensorial). El observador percibe objetos que atraen su atención, y forma juicios de valor de lo que ve.

Por último, los datos disponibles indican para el arte rupestre de Ventania un rango cronológico aproximado desde 1500 años AP basado en un fechado sobre restos faunísticos asociados estratigráficamente a un fragmento con pintura hasta momentos del contacto hispano-indígena, por la presencia de un motivo de embarcación en el sitio Cueva Florencio (Oliva 2000). ${ }^{1}$

\section{ESTUDIO SEMIÓTICO}

Las representaciones rupestres de Ventania son consideradas como un sistema estructurado de comunicación gráfica, que habría actuado en el procesamiento de información, para transmitir o almacenar conocimiento. Como en un sistema semiótico cada imagen es un signo con un significado simbólico, y estos signos se combinan en patrones reconocibles utilizando reglas, se buscó identificar la recurrencia de los signos y sus patrones de asociación para obtener una idea de cómo trabaja el sistema. Las cualidades estéticas anteriormente abordadas se refieren al conjunto de formas, estimulaciones sensoriales y relaciones espaciales que permiten que estas pinturas sean apropiadas en su contexto cultural, y configuran el criterio estético particular y específico de una determinada sociedad, sobre el cual se basa el sistema semiótico que se materializa en las pinturas.

Para realizar este análisis se aplica una metodología (Magariños 1996, 2003 y 2004) que se encuadra dentro de la semiótica general, que es aquella que desarrolla los conceptos de Peirce (1965) y de Saussure (1972). En el marco de la semiótica visual (específicamente Magariños 1999), se identificaron en las representaciones rupestres abstracto-geométricas analizadas, las marcas (unidades mínimas) y los atractores (combinación de estas unidades) presentes. Este análisis permite al investigador actual realizar una aproximación a la estructura cognitiva del grupo que realizó estas imágenes en el pasado, a las reglas de combinación que rigieron su elaboración, a partir de los elementos básicos de diseño. El arte rupestre como combinación semiótica obliga al observador a la búsqueda de los códigos de reconocimiento para describir y descodificar su contenido. La operación de reconocimiento se articula en el sistema cultural de quien lo percibe, ya que una imagen puede ser entendida desde la perspectiva del observador.

\section{Marcas}

Se entiende por "marca" la mayor porción de imagen cuya percepción todavía no actualiza un atractor existencial, en cuanto es una parte que no posee significación por sí sola, pero que la adquirirá al integrarse sintácticamente con otra u otras (Magariños 2008: 228).

En las representaciones rupestres de Ventania se han registrado un total de ocho marcas, las cuales fueron categorizadas como: línea recta, línea quebrada (u ortogonal), línea en V o zigzag, triángulo, rombo, rectángulo (forma subrectangular), punto y elipse (forma subcircular). 


\section{Atractores}

Se entiende por "atractor" de una imagen material visual a un conjunto de formas que, en un momento dado, se organiza, con cierta constancia, en una imagen mental almacenada en la memoria visual, la cual se actualiza o no por su correspondencia o falta de correspondencia con la configuración que el perceptor efectúa a partir de determinada imagen material visual propuesta (Magariños 2008: 226).

Dentro del corpus de arte rupestre de Sierra de la Ventana fueron identificados algunos atractores icónicos compuestos por la combinación de marcas de carácter geométrico (círculos, rectángulos, líneas rectas, entre otros). Estos atractores fueron clasificados en antropomorfos, estos a su vez se dividen en esquemáticos y mascariformes; biomorfos, manos positivas, y una embarcación. Excepto en un sitio (Gruta de los Espíritus) donde solo se hallan representados mascariformes, el resto de los atractores icónicos aparecen en sitios que presentan también atractores no icónicos (fig. 4). Estos atractores icónicos no se incluyen en el análisis presentado en este trabajo, motivo por el cual no se desarrollarán otros aportes en este sentido.
El 80\% de los sitios con arte rupestre relevados presenta atractores exclusivamente no icónicos (ejemplo Cueva 2 Parque Tornquist). El 16\% de los sitios presenta ambos tipos de atractores (ejemplo Cueva 1 Arroyo Cochenleufú Chico). Los atractores no icónicos identificados en las pinturas de Ventania se clasificaron como: líneas aisladas, líneas paralelas, líneas en V, líneas entrecruzadas, líneas curvas, líneas en zigzag, líneas ortogonales, guardas en $\mathrm{V}$, guardas de triángulos en posición normal e invertida, triángulos unidos, guardas de triángulos llenos, guarda en rombos, escalonados, elipse, puntiformes, cruciformes, circunferencias y alineamiento de puntos.

\section{Operaciones combinatorias}

La segmentación de los atractores permitió detectar cinco operaciones combinatorias empleadas en la configuración de las representaciones rupestres de Ventania: combinación simple, repetición, superposición, concentricidad y simetría bilateral. Asimismo, debe señalarse que existen casos en los cuales no se ha utilizado ninguna operación combinatoria, el ejemplo más claro lo constituye la línea recta aislada. Por otra parte, cabe destacar que la mayoría de los atractores identificados

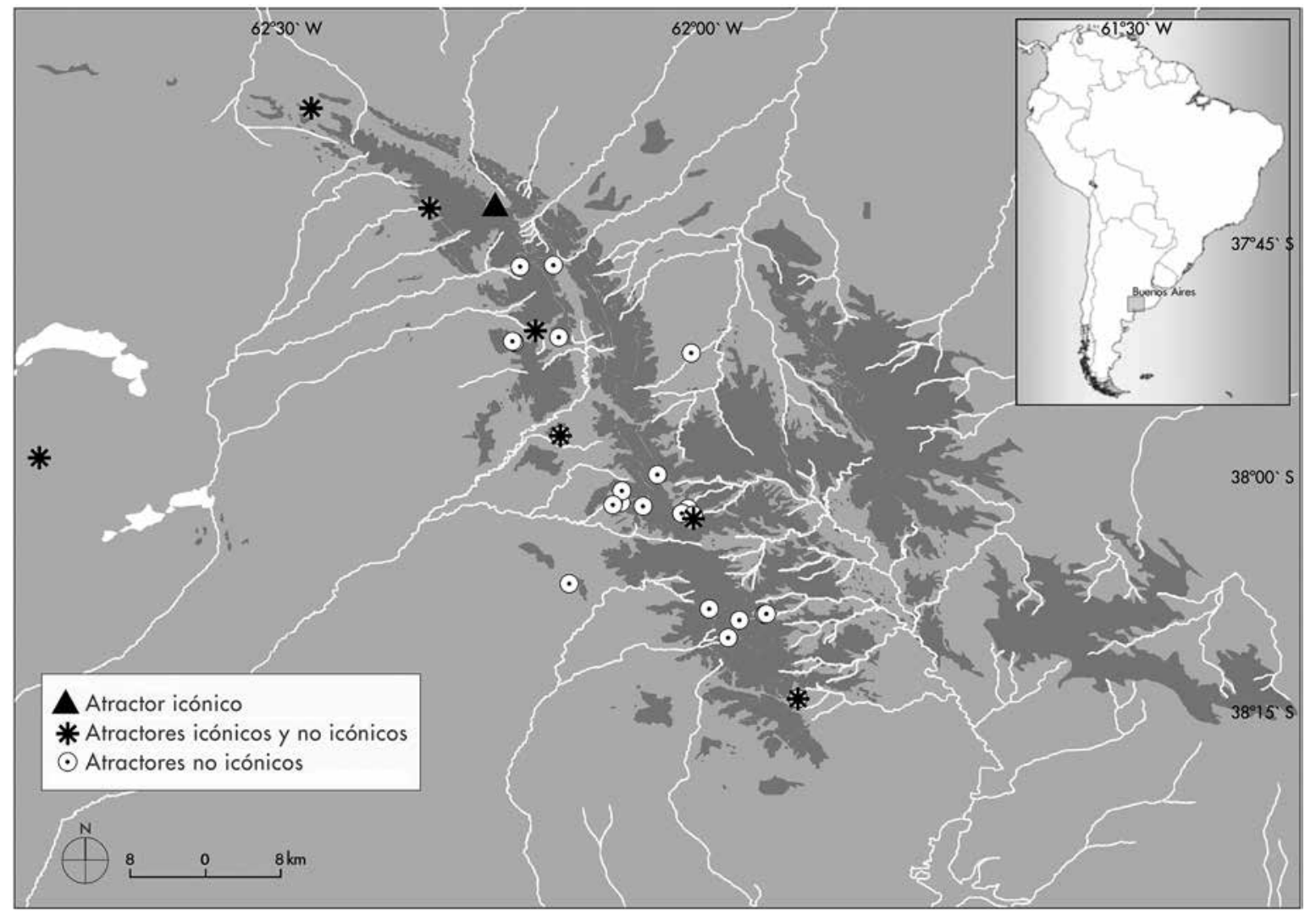

Figura 4. Distribución de los atractores correspondientes a los sitios con arte rupestre de Ventania. Figure 4. Distribution of attractors corresponding to the rock ark sites of Ventania. 
se encuentran aislados en la composición del panel de arte. De las operaciones combinatorias mencionadas, la repetición es la más utilizada, ya que aparece en veintinueve de los sitios, generalmente para originar el atractor de líneas paralelas.

La información precedente, obtenida mediante el análisis semiótico, se encuentra sintetizada en la Tabla 1.

Aunque a primera vista pueda parecer que las categorías aplicadas (marca, atractor, operación combinatoria) inducen a error porque no son mutuamente excluyentes entre sí, debe aclararse que la marca es siempre singular (i. e. triángulos enfrentados, guarda de triángulos), excepto en el caso de la línea recta aislada, que no indica ninguna operación combinatoria y su determinación es contextual. El plural utilizado en los atractores definidos no es casual, dado que la repetición es la operación combinatoria empleada en la mayoría de los sitios, como fue mencionado precedentemente.

\section{REPRESENTACIONES VISUALES DE ELEMENTOS PORTÁTILES DE VENTANIA}

En sitios arqueológicos del área de Ventania se han hallado diferentes elementos portátiles que presentan algún tipo de diseño en su superficie, correspondientes al Holoceno Tardío (Oliva 2006), coincidiendo con lo expresado previamente para las cuevas y aleros con pinturas rupestres. Se ha recuperado cerámica decorada en los sitios Laguna Gascón 1, Los Chilenos 1 y 2, y La Montaña 1; y placas grabadas en Los Chilenos 2, y La Montaña 1 (Barrientos et al. 1997; Oliva 2006). Asimismo, se han estudiado fragmentos cerámicos con decoración pertenecientes a los museos de Chasicó, Saavedra y Puan, además de hematitas y placas grabadas de los sitios Ybarra y La Escondida depositadas en el Museo de Chasicó.

Respecto de la decoración cerámica, la alfarería temprana de la provincia de Buenos Aires presenta motivos decorativos incisos, los diseños son geométricos (Caggiano et al. 2001). En el centro oeste y sur bonaerense los motivos decorativos pueden ser semicírculos combinados con líneas incisas paralelas e incisos por arrastre; otros diseños geométricos de la incisión son líneas rectas, curvas, puntos; triángulos, cuadrados y rectángulos rellenos de líneas punteadas en su interior; los motivos combinan zigzag con línea continua, líneas verticales y horizontales. Pueden estar asociados con pintura roja (Caggiano et al. 2001). Los restos de alfarería de los sitios del sistema serrano de Ventania y su llanura adyacente presentan decoración incisa y restos de pintura roja. La decoración presenta motivos geométricos simples y complejos (Catella 2004). Los sitios del área de estudio donde se ha hallado material cerámico pueden ser asignados al período final del precontacto, o bien a comienzos del período hispano-indígena, excepto Laguna Gascón, cuyo contexto arqueológico puede atribuirse plenamente al período de contacto hispano-indígena (Catella 2004; Oliva et al. 2007). El material cerámico del área de estudio proviene de las colecciones depositadas en los museos de Chasicó, Saavedra y Puan, y de los sitios Laguna Gascón 1, Los Chilenos 1 y 2 y La Montaña 1.

En cuanto a las placas grabadas, estas han sido recuperadas tanto en superficie -como es el caso de aquella perteneciente al sitio Ybarra en proximidades del río Sauce Chico, depositada en el Museo de Chasicó- como en estratigrafía, por medio de excavaciones sistemáticas, en los sitios Los Chilenos 2, en inmediaciones de la laguna homónima, y La Montaña 1, ambos con una ocupación inmediatamente anterior a la conquista hispánica (Barrientos et al. 1997; Oliva 2000). Cabe destacar que en todos los casos las placas presentan motivos incisos compuestos por atractores no icónicos, geométricos rectilíneos (Oliva \& Algrain 2004).

Otro registro comparable consiste en dos huevos de ñandú hallados en la Laguna del Monte de la localidad de Guaminí, que presentan a lo largo de sus ejes centrales una guarda de líneas paralelas de $2 \mathrm{~cm}$ de ancho (Oliva 2006). Los motivos de líneas paralelas se observan en la mayoría de los sitios en cuevas y aleros del sistema serrano de Ventania. Además, puede mencionarse la descripción realizada por Vignati (1937, 1938) de un cráneo pintado con motivos geométricos en varios colores, de un sitio en la península de San Blas, y se relaciona con otros hallazgos de entierros secundarios efectuados en la región pampeana que presentan huesos con evidencias de pintura roja, entre los que se encuentran los pertenecientes al sitio Laguna Los Chilenos 1 (Barrientos et al. 1997).

Respecto de la evidencia etnográfica disponible, pueden mencionarse los quillangos (cueros utilizados en momentos de contacto hispano-indígena por los grupos indígenas de La Pampa y la Patagonia), ya que Oliva y colaboradores (Oliva \& Algrain 2004) han observado semejanzas entre los motivos abstractos de cueros de guanaco, vaca, caballo y zorro depositados en el Museo de Ciencias Naturales de La Plata, y algunas de las pinturas documentadas en cuevas y aleros del sistema serrano de Ventania.

Los resultados obtenidos se sintetizan en una tabla comparativa entre los diversos soportes de las representaciones visuales respecto de los atractores no icónicos, indicando, además, su presencia o ausencia en los diferentes soportes (Tabla 2). 
Tabla 2. Cuadro con la presencia de atractores no icónicos en distintos tipos de soporte arqueológico en el área del sistema serrano de Ventania y su llanura adyacente.

Table 2. Presence of non-iconic attractors on different types of archeological supports in the serrano system area of Ventania and its adjacent plains.

\begin{tabular}{|c|c|c|c|c|c|}
\hline Atractores no icónicos & Arte rupestre & $\begin{array}{l}\text { Placas } \\
\text { grabadas }\end{array}$ & Cerámica & $\begin{array}{c}\text { Otros } \\
\text { elementos } \\
\text { simbólicos }\end{array}$ & $\begin{array}{l}\text { Elementos } \\
\text { etnográficos }\end{array}$ \\
\hline Líneas aisladas & $\mathrm{X}$ & & & & \\
\hline Líneas paralelas & $\mathrm{X}$ & $\mathrm{X}$ & $\mathrm{X}$ & $\mathrm{X}$ & $\mathrm{X}$ \\
\hline Líneas en V & $\mathrm{X}$ & & & $\mathrm{X}$ & $\mathrm{X}$ \\
\hline Líneas entrecruzadas & $\mathrm{X}$ & $\mathrm{X}$ & $\mathrm{X}$ & & \\
\hline Líneas curvas & $\mathrm{X}$ & & & $\mathrm{X}$ & \\
\hline Líneas en zigzag & $\mathrm{X}$ & $\mathrm{X}$ & & $\mathrm{X}$ & $\mathrm{X}$ \\
\hline Líneas ortogonales & $\mathrm{X}$ & & & & $\mathrm{X}$ \\
\hline Guardas en V & $\mathrm{X}$ & $\mathrm{x}$ & & $\mathrm{X}$ & $\mathrm{X}$ \\
\hline $\begin{array}{c}\text { Guardas de triángulos en posición } \\
\text { normal e invertida }\end{array}$ & $\mathrm{X}$ & $\mathrm{X}$ & $\mathrm{X}$ & & $\mathrm{X}$ \\
\hline Triángulos unidos & $\mathrm{X}$ & $\mathrm{X}$ & & & $\mathrm{X}$ \\
\hline Guardas de triángulos llenos & $\mathrm{x}$ & & & & $\mathrm{X}$ \\
\hline Guarda en rombos & $\mathrm{X}$ & $\mathrm{X}$ & $\mathrm{X}$ & & $\mathrm{X}$ \\
\hline Escalonados & $\mathrm{X}$ & & $\mathrm{X}$ & & \\
\hline Elipse & $\mathrm{X}$ & & & & \\
\hline Puntiformes & $\mathrm{X}$ & & & & \\
\hline Cruciformes & $\mathrm{X}$ & & & & $\mathrm{X}$ \\
\hline Circunferencias & $\mathrm{X}$ & & & & \\
\hline Alineamiento de puntos & $\mathrm{X}$ & & & & \\
\hline Indeterminado & $\mathrm{X}$ & & & $\mathrm{X}$ & \\
\hline
\end{tabular}

Es destacable la aparición de determinados atractores (líneas paralelas, guarda de rombos, guarda de triángulos, líneas en zigzag, guardas en $\mathrm{V}$, entre otros) recurrentemente en los distintos tipos de soportes (arte rupestre, cerámica, placas grabadas, otros), lo que estaría vinculando las manifestaciones visuales indígenas tardías y un código común que subyace en la visión de mundo de sus hacedores. La ejecución de motivos abstractos rectilíneos, líneas paralelas, zigzags, rectas cruzadas en ángulos agudos, entre otros, se encuentra presente en distintos soportes (paredes rocosas, placas, cerámica, cueros, entre otros). Esta variabilidad de soportes con representaciones similares (fig. 5) marca una codificación compartida en la representación de las sociedades indígenas en momentos asociados al Período Tardío (Oliva 2006), pero que podría tener antecedentes más antiguos en áreas vecinas, lo que indicaría un caso de perduración de símbolos cuya significación se habría modificado al cambiar de contexto, es decir, se trataría de objetos resignificados.

Asimismo, se pudo observar que el arte rupestre del sistema de Ventania se vincula directamente con representaciones localizadas en otros puntos de la región pampeana, como en las sierras de Tandilia y Lihue Calel (Tabla 3). Se registró una estética abstracta geométrica compartida presente en los sitios ubicados en la subregión Pampa Seca (Schatzky 1954; Zetti \& Casamiquela 1967; Gradín 1975; Schobinger \& Gradín 1985; Aguerre 2000; Curtoni 2007); en la subregión Pampa Húmeda (Ceresole \& Slavski 1985; Arana \& Mazzanti 1987; Mazzanti 1991; Mazzanti \& Valverde 1997, 2003; Madrid et al. 2000) y en el sur del Área Ecotonal Húmedo-Seca Pampeana. Este fenómeno estaría relacionado con la tendencia abstracta geométrica compleja postulada inicialmente por Gradín (1997-1998) y que está siendo reformulada por Podestá y colaboradores (2011). Esta similitud sin dudas 


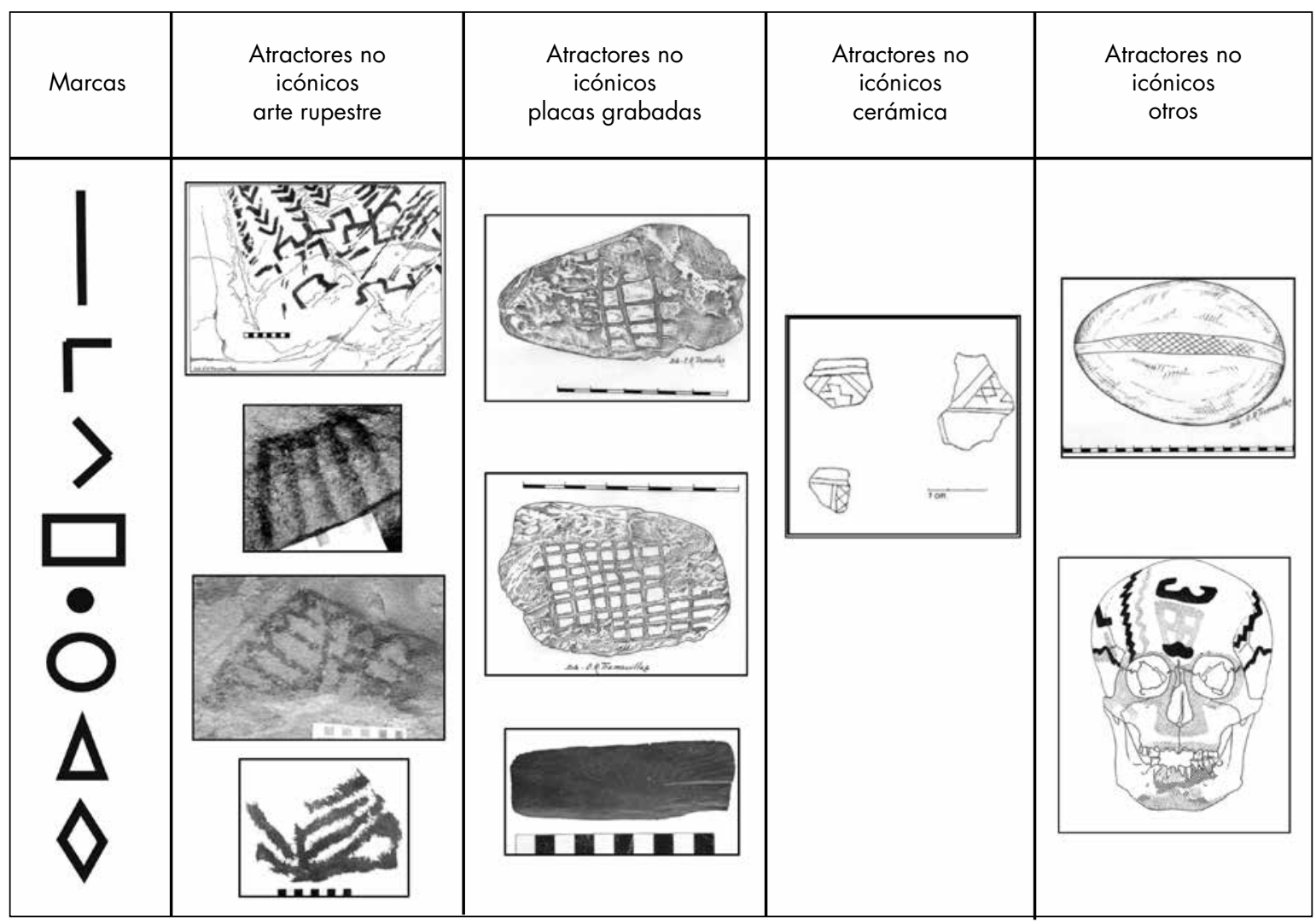

Figura 5. Cuadro comparativo con las marcas utilizadas para la configuración de atractores no icónicos en diferentes soportes presentes en el registro arqueológico.

Figure 5. Comparison of marks used for the configuration of non-iconic attractors on different supports present in the archeological record.

está indicando determinadas recurrencias de motivos que deben entenderse dentro de procesos sociales de escala macrorregional.

\section{CONSIDERACIONES FINALES}

Las expresiones plásticas son un producto de un contexto cultural e históricamente dado, y se encuentran sujetas a códigos estéticos, preferencias sociales y valoraciones propias de una sociedad.

Cierta estandarización presente en las representaciones abstractas geométricas del área, principalmente en el arte rupestre, pero también en otros soportes, como placas grabadas y cerámica, implicaría la presencia de un código estético subyacente a la creación y reproducción de estos diseños. Esta repetición de determinados diseños habría permitido la codificación de mensajes o significados según el código simbólico mencionado, asegurando la transmisión efectiva del mensaje. En síntesis, se observó una marcada preferencia por la utilización de signos no icónicos dentro del vocabulario gráfico correspondiente a los grupos cazadores recolectores que habitaron el área de Ventania.

Dentro de este corpus, determinados atractores no icónicos, como las líneas paralelas, ortogonales y en zigzag, junto con los triángulos unidos por el vértice, los puntiformes y subcirculares, adquieren una relevancia particular por la cantidad y amplia dispersión por la región pampeana, en tanto las líneas aisladas, las guardas en $\mathrm{V}$ y los cruciformes habrían conformado una identidad local propia del área de Ventania. Esta identidad local también estaría vinculada con la generación de categorías diferentes de mensajes según los destinatarios (público/ privado), que servirían para configurar sitios portadores de mensajes públicos y otros lugares con restricción parcial o total en el proceso de transmisión del mensaje (privado), causando efectos distintos en el observador.

En este sentido, la dimensión estética de las pinturas constituye solo una de las líneas posibles para abordar su estudio, sin aislarlas de los otros aspectos de la cultura material para contextualizar estas representaciones en el marco de la sociedad que las produjo. La perspectiva estética está orientada a la materialidad de la obra, a la 
Tabla 3. Cuadro con la presencia de atractores no icónicos en el arte rupestre de tres zonas ecológicas diferentes de la región pampeana: Pampa Seca (provincia de La Pampa), Área Ecotonal Húmedo Seca Pampeana (AEHSP: Ventania)

y Pampa Húmeda (Tandilia), estas dos últimas en la provincia de Buenos Aires.

Table 3. Presence of non-iconic attractors in the rock art of three different ecological zones of the Pampean Region:

Dry Pampa (La Pampa Province), Wet-Dry Pampa Ecotone (AEHSP: Ventania), and Wet Pampa (Tandilia), the latter two in Buenos Aires Province.

\begin{tabular}{|c|c|c|c|}
\hline Atractores no icónicos & Pampa seca & AEHSP & Pampa húmeda \\
\hline Líneas aisladas & & $\mathrm{X}$ & \\
\hline Líneas paralelas & $\mathrm{X}$ & $\mathrm{X}$ & $\mathrm{X}$ \\
\hline Líneas en $\mathrm{V}$ & & $\mathrm{X}$ & $\mathrm{X}$ \\
\hline Líneas entrecruzadas & & $\mathrm{X}$ & $\mathrm{X}$ \\
\hline Líneas rectilíneas & $\mathrm{X}$ & $\mathrm{X}$ & $\mathrm{X}$ \\
\hline Líneas curvas & $\mathrm{X}$ & $\mathrm{X}$ & $\mathrm{X}$ \\
\hline Líneas en zigzag & $\mathrm{X}$ & $\mathrm{X}$ & $\mathrm{X}$ \\
\hline Líneas ortogonales & $\mathrm{X}$ & $\mathrm{X}$ & $\mathrm{X}$ \\
\hline Guardas en V & & $\mathrm{X}$ & \\
\hline Guardas de triángulos en posición normal e invertida & & $\mathrm{X}$ & $\mathrm{X}$ \\
\hline Triángulos unidos por el vértice (clepsidra) & $\mathrm{X}$ & $\mathrm{X}$ & $\mathrm{X}$ \\
\hline Guardas de triángulos llenos & & $\mathrm{X}$ & $\mathrm{X}$ \\
\hline Guarda en rombos & $\mathrm{X}$ & $\mathrm{X}$ & \\
\hline Elipse & & $\mathrm{X}$ & $\mathrm{X}$ \\
\hline Puntiformes & $\mathrm{X}$ & $\mathrm{X}$ & $\mathrm{X}$ \\
\hline Cruciformes & & $\mathrm{X}$ & \\
\hline Circunferencias y subcirculares & $\mathrm{X}$ & $\mathrm{X}$ & $\mathrm{X}$ \\
\hline Alineamiento de puntos & $\mathrm{X}$ & $\mathrm{X}$ & \\
\hline Enmarcados & & $\mathrm{X}$ & $\mathrm{X}$ \\
\hline Reticulados & & $\mathrm{X}$ & $\mathrm{X}$ \\
\hline U acostada & $\mathrm{X}$ & & \\
\hline Escalonados, líneas quebradas & $\mathrm{X}$ & & $\mathrm{X}$ \\
\hline Indeterminado & $\mathrm{X}$ & $\mathrm{X}$ & $\mathrm{X}$ \\
\hline
\end{tabular}

forma de la representación visual; en cambio, el enfoque semiótico busca trascender la apariencia, con el fin de hallar el contenido, es decir, comprender el significado. Sin embargo, ambas concuerdan en un aspecto fundamental: la característica comunicacional de las representaciones, y es justamente a través de la comunicación de un mensaje que se buscó, desde la semiótica, construir el vocabulario de signos utilizados, y a través de la estética, indagar en los efectos de este mensaje.

Si bien se accede a este tipo de representaciones visuales desde una mirada condicionada culturalmente, ya que pertenece a una sociedad cuya visión de mundo y sistema de representación está desvinculada de esas pinturas, se considera posible entender el arte rupestre como evidencia arqueológica que puede aportar conocimiento acerca de los cambios culturales y sociales durante el Holoceno, constituyendo un agente activo en los procesos mencionados. Las representaciones rupestres implican la existencia de un código que permite la comunicación, pero también un espacio significativo en el cual adquieren sentido las dinámicas socioculturales. Por lo tanto, resulta sumamente relevante el aporte que puede proporcionar la utilización de un enfoque semiótico en el análisis de las pinturas, por estar orientado a explicar la producción del significado de los fenómenos sociales, lo cual contribuiría a la generación de datos dentro de la dimensión interpretativa, que resulta la más complicada de desarrollar para los arqueólogos. 


\section{NOTAS}

1 Se fecharon restos óseos apendiculares de Lama guanicoe, hallados en contexto estratigráfico en la cueva La Sofía 4, asociado a elementos líticos y a un fragmento de roca cuarcítica de iguales características físicas que las paredes y techo de la cueva. Este fragmento evidenciaba en uno de sus lados pintura roja desvaída, similar a las pinturas rupestres presentes en la cueva, consistentes en una concentración de motivos de líneas paralelas y motivos aislados en color rojo desvaído en la pared del sector suroeste. La datación dio por resultado $1595 \pm 70$ años AP (Oliva 2000).

\section{REFERENCIAS}

Aguerre, A. M., 2000. Las pinturas rupestres de Chos Malal. Meseta basáltica del oeste de la provincia de La Pampa. En Arte en las rocas. Arte rupestre, menhires y piedras de colores en Argentina, M. M. Podesta \& M. de Hoyos, Eds., pp. 135-142. Buenos Aires: Sociedad Argentina de Antropología.

Arana, M. \& D. Mazzanti, 1987. Manifestaciones de arte rupestre en el Pdo. De General Pueyrredón. Historia Regional Bonaerense. En Actas de las I, II y III Jornadas de Historia Regional Bonaerense, Tomo 2, pp. 147-149. Tandil: UNCPBA.

Barrientos, G.; M. Leipus \& F. Oliva, 1997. Investigaciones arqueológicas en la laguna Los Chilenos (provincia de Buenos Aires). En Arqueología Pampeana en la década de los '90, M. Berón \& G. Politis, Eds., pp. 115-125. San Rafael, Mendoza: Museo Municipal de Historia Natural de San Rafael e INCUAPA.

Caggiano, M. A.; V. H. Garay \& C. Moreyra, 2001. Iconografía bonaerense. Alfarería Prehispánica. La Plata: Ed. Hombre Barro Fuego.

CATElla, L., 2004. Análisis tecnológico del material cerámico del sistema serrano de Ventania y llanura adyacente (provincia de Buenos Aires). En Miradas. Trabajos de las V Jornadas de Jóvenes Investigadores en Ciencias Antropológicas, M. Carballido, C. Pissarello \& A. Re, Eds., pp. 170-183. Buenos Aires: Instituto Nacional de Antropología y Pensamiento Latinoamericano. CD-Rom.

Ceresole, G. \& J. Slavsky, 1985. Informe preliminar sobre la localidad Lobería 1 (provincia de Buenos Aires). En Resúmenes de los Trabajos a Presentarse (Comunicaciones y Ponencias) en VIII Congreso Nacional de Arqueología Argentina, p. 4, Concordia

Consens, M. \& F. Oliva, 1999. Estado de las investigaciones en sitios con representaciones rupestres en la región pampeana, República Argentina. En Actas del XI Congreso Nacional de Arqueología Argentina, C. Diez Marín, Ed., pp. 218-228. La Plata: Facultad de Ciencias Naturales y Museo, UNLP.

Curtoni, R., 2007. El paisaje y las expresiones rupestres de los cazadores recolectores pampeanos de Argentina. International Journal of South American Archaeology 1: 40-48.

DetTwiler, A., 1986. Análisis del arte rupestre, entre la miopía funcionalista y el imperialismo de la semiótica. Chungara 16-17: 451-458.

Eco, U., 1995 [1976]. Tratado de semiótica general. Barcelona: Editorial Lumen.

Gradín, C. J., 1975. Contribución a la arqueología de La Pampa (Arte rupestre). Santa Rosa, Dirección Provincial de Cultura: Manzini Hnos.

- 1997-1998. El arte rupestre del sur mendocino entre los siglos VIII y XV de la era. ¿Un área de conflicto o de convivencia? Relaciones de la Sociedad Argentina de Antropología XXIIXXIII: 7-23.

HeYD, T., 2001. El arte rupestre y la apreciación estética de paisajes naturales. Relaciones de la Sociedad Argentina de Antropología XXVI: 397-402.

- 2005. Aesthetics and Rock Art: an introduction. In Aesthetics and Rock Art, T. Heyd \& J. Clegg, Eds., pp. 1-19. Aldershot: Ashgate.
Madrid, P. \& F. OlIVA, 1994. Análisis preliminar de las representaciones rupestres presentes en cuatro sitios del sistema de Ventania, provincia de Buenos Aires. Revista del Museo de La Plata: 199-223.

Madrid, P.; G. Politis \& D. Poiré, 2000. Pinturas rupestres y estructuras de piedras en las sierras de Curicó (extremo noroccidental de Tandilia, región pampeana). Intersecciones en Antropología 1: 35-53.

Magariños, J., 1996. Fundamentos lógicos de la semiótica y su práctica. Buenos Aires: Ed. Edicial.

— 1999. Operaciones semióticas en el análisis de historietas. En Fronteras de la semiótica. Homenaje a Desiderio Blanco, O. Quezada Macchiavello, Ed., pp. 433-446. Perú: Universidad de Lima/Fondo de Cultura Económica.

- 2003. Hacia una semiótica indicial. La Coruña: Ediciós Do Castro

- 2004. Los 4 signos. Diseño de las operaciones fundamentales en metodología semiótica. Razón y palabra 38. [Online] <http:// www.razonypalabra.org.mx/anteriores/n38/jmagarinos.html> [Citado 25-09-2007].

— 2008. La semiótica de los bordes. Apuntes de metodología semiótica. Córdoba: Ed. Comunicarte.

MazzanTi, D., 1991. Haras Los Robles: un sitio con pictografías en el borde oriental de las Sierras de Tandilia. Boletín del Centro 3: 180-200, La Plata.

Mazzanti, D. \& F. VAlverde, 1997. Nuevos sitios arqueológicos con representaciones rupestres en las sierras de Tandilia Oriental (provincia de Buenos Aires). En Actas del XII Congreso Nacional de Arqueología Argentina, Tomo II, C. Diez Marín, Ed., pp. 390. La Plata: Facultad de Ciencias Naturales y Museo UNLP. - 2003. Representaciones rupestres de cazadores-recolectores en las sierras de Tandilia oriental: una aproximación a la arqueología del paisaje. En Actas del XIII Congreso Nacional de Arqueología Argentina, tomo III, pp. 311-316, Córdoba.

Morphy, H., 1993. For the Motion (1). En Key Debates in Anthropology, T. Ingold, Ed., pp. 255-260. Londres: Routledge.

1994. The Anthropology of Art. En Companion Encyclopedia to Anthropology, T. Ingold, Ed., pp. 648-685. Londres: Routledge.

Oliva, F., 2000. Análisis de las localizaciones de los sitios con representaciones rupestres en el sistema serrano de Ventania, provincia de Buenos Aires. En Arte en las rocas. Arte rupestre, menhires y piedras de colores en Argentina, M. M. Podestá \& M. de Hoyos, Eds., pp. 143-158. Buenos Aires: Sociedad Argentina de Antropología.

— 2006. Uso y contextos de producción de elementos "simbólicos" del sur y oeste de la provincia de Buenos Aires, República Argentina (área Ecotonal Húmedo-Seco Pampeana). Revista de la Escuela de Antropología XII: 101-115. Rosario: Escuela de Antropología de la Universidad Nacional de Rosario.

Oliva, F. \& M. Algrain, 2004. Una aproximación cognitiva al estudio de las representaciones rupestres del Casuhati (sistema serrano de Ventania y llanura adyacente, provincia de Buenos Aires) En La región rampeana. Su pasado arqueológico, C. Gradín \& F. Oliva, Eds., pp. 49-60. Rosario: Editorial Laborde.

- 2005. Representaciones simbólicas de las sociedades indígenas en el Área Ecotonal Húmedo-Seca Pampeana (AEHSP) ¿Arte shamánico? Revista de la Escuela de Antropología X: 155-168. Rosario: Escuela de Antropología, Universidad Nacional de Rosario.

Oliva, F.; M. Algrain \& M. C. Panizza, 2010. Diferentes enfoques en la investigación del arte rupestre del sistema serrano de Ventania. Comechingonia 13: 89-107. Córdoba: Universidad Nacional de Córdoba.

Oliva, F.; G. L'Heureux, H. De Angelis, V. Parmigiani \& F. Reyes. 2007. Poblaciones indígenas de momentos postcontacto en el borde occidental de la pampa húmeda: Gascón 1, un sitio de entierros humanos. En Arqueología argentina en los inicios de un nuevo siglo, F. Oliva, N. de Grandis \& J. Rodriguez, Eds., pp. 265-274. Rosario: Laborde Libros Editor. 
Peirce, C. S., 1965. Collected Papers. Cambridge: The Belknap Press of Harvard University Press.

Pérez AMAT, M.; D. SCHeINes \& M. BAYÓN, 1985. Noticia preliminar sobre hallazgos de pinturas rupestres en el establecimiento Santa Marta (Partido de Saavedra). Sapiens 5: 86-90. Chivilcoy.

PODESTÁ, M. M; F. OlIVA \& A. Florines, 2011. Indicadores estilísticos de interacción a través del arte rupestre de Pampa-Patagonia y Cuenca del Plata (Argentina-Uruguay). En Actas I Congreso Internacional de Arqueología de la Cuenca del Plata, IV Encuentro de Discusión Arqueológica del Nordeste Argentino y las II Jornadas de Actualización en Arqueología Tupiguaraní, pp. 175-176. Buenos Aires.

Saussure, F. DE, 1972 [1915]. Cours de Linguistique Générale. París: Payot.

SCHATZKY, I., 1954. Las pictografías de Lihuel-Calel. Revista Geográfica Americana: 85-89.

SCHILLER, W., 1930. Investigaciones geológicas en las montañas del sudoeste de la Provincia de Buenos Aires. En Anales del Museo de La Plata IV: 11-96. La Plata.
Schobinger, J. \& C. Gradín, 1985. Arte Rupestre de la Argentina. Cazadores de la Patagonia y agricultores andinos. Madrid: Encuentro Ediciones.

Suero, T., 1972. Compilación geológica de las Sierras Australes de la Provincia de Buenos Aires. División Geología - LEMIT. Provincia de Buenos Aires. Anales 3: 135-147. La Plata.

Troncoso, A., 2005. Hacia una semiótica del arte rupestre de la cuenca superior del río Aconcagua, Chile Central. Chungara 37 (1): 21-35.

Vignati, M. A., 1937. Origen étnico de los cráneos pintados de San Blas. Relaciones de la Sociedad Argentina de Antropología 1: 52-57.

— 1938. Cráneos pintados del cementerio indígena de San Blas. Revista del Museo de La Plata 1: 35-52.

Zetti, J. \& R. CASAmiquela, 1967. Noticia sobre una breve expedición arqueológica a la zona de Lihue Calel (provincia de La Pampa) y observaciones complementarias. Cuadernos del Sur: 3-40. Bahía Blanca: Instituto de Humanidades de la Universidad del Sur. 
